\title{
Midkine is involved in the pathogenesis of impaired osteoporotic fracture healing after ovariectomy in mice
}

Haffner-Luntzer M. ${ }^{1}$, Kemmler J. ${ }^{1}$, Prystaz K. ${ }^{1}$, Heidler V. ${ }^{1}$, Schinke T. ${ }^{2}$, Amling M. ${ }^{2}$, Ignatius A. ${ }^{1}$, Liedert A. ${ }^{1}$

${ }^{1}$ Institute of Orthopaedic Research and Biomechanics, Ulm University, Germany

2Institute of Osteology and Biomechanics, University Medical Center Hamburg-Eppendorf, Germany

melanie.haffner-luntzer@uni-ulm.de

\section{Objectives}

Clinical data demonstrated significantly impaired bone regeneration in postmenopausal osteoporotic patients [1]. The molecular mechanisms behind that are still unclear. Therefore, there is a high clinical need for new treatment strategies.

One promising drug target molecule is the heparin-binding growth- and differentiation factor Midkine (Mdk), because:

$>$ Mdk is supposed to be a negative regulator of bone formation [2]

$>$ Mdk negatively affects Wnt-signaling and therefore osteogenic differentiation in osteoblasts [3,4]

$>$ Antagonizing systemic Mdk accelerated bony callus formation during fracture healing [4]

$>$ Mdk is an estrogen responsive gene with increased expression in the postmenopausal, diabetic kidney [5]

Is Mdk involved in delayed osteoporotic fracture healing?

\section{Methods}

Animal model: 3-months-old female wildtype mice (C57BL/6J)

Surgery: Bilateral ovariectomy (Fig. 1A); 4 weeks later: standardized femur osteotomy stabilized with an external fixator (Fig. 1B, C)

Treatment: Injections with $25 \mathrm{mg} / \mathrm{kg}$ BW Mdk-antibody (Mdk-Ab) or vehicle 2x/week for 3 weeks

Analyses: 3-point-bending test (Fig. 1D) and $\mu \mathrm{CT}$ (Fig. 1E) at day 23; histomorphometry and immunohistochemistry at day 10 and 23; Mdk serum ELISA at day 3,10 and 23.

Statistics: Kruskal-Wallis test with Dunn's post hoc $\left(n=6-7 ;{ }^{*} p<0.05\right)$.

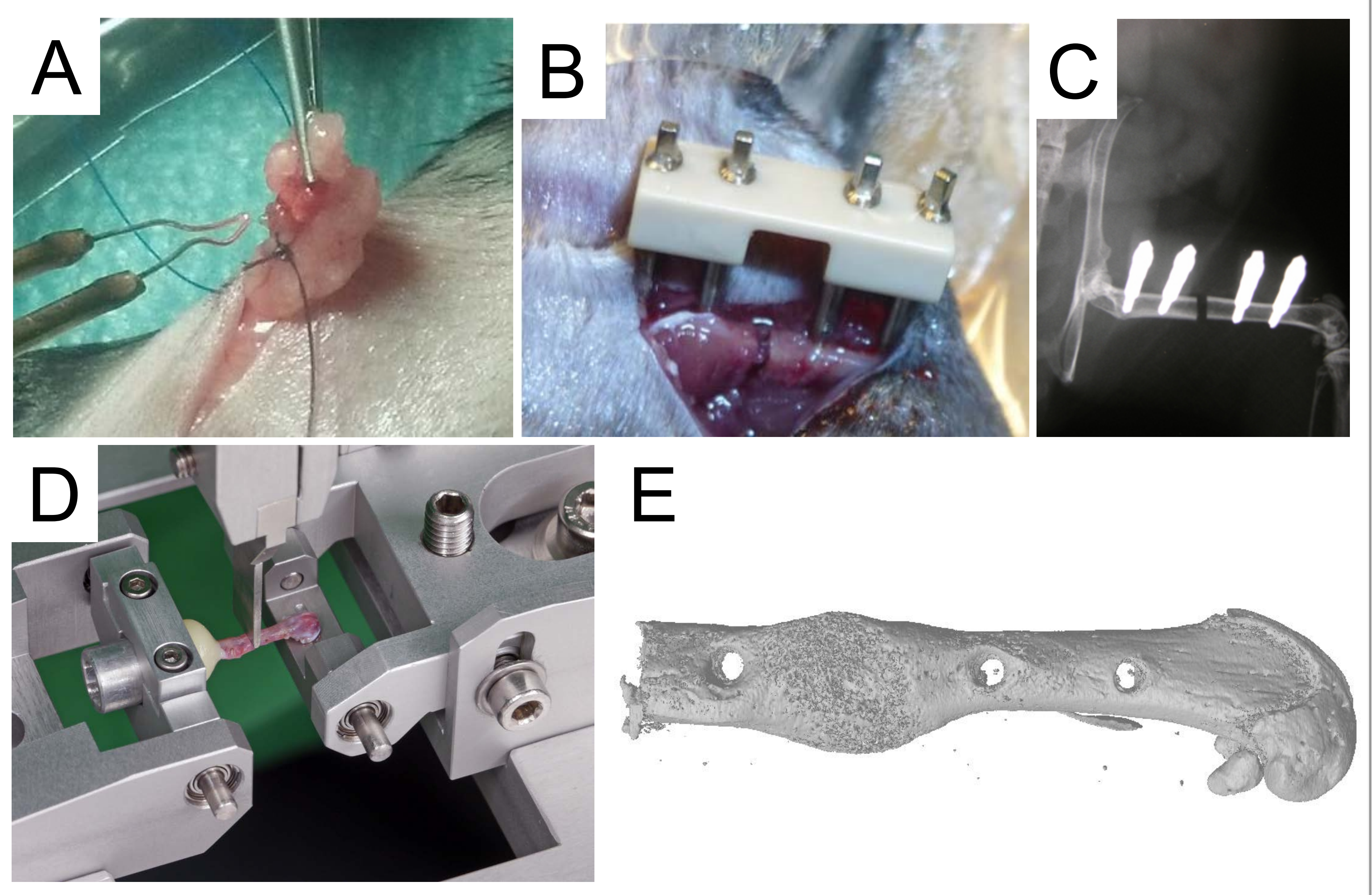

Fig. 1: A) Ovariectomy of female mouse B) Mouse with an external fixator at the right femur directly after sawing of the osteotomy C) X-ray of a mouse with a femoral fracture stabilized by an external fixator $D)$ Non-destructive 3-point bending test $E$ ) $3 D \mu C T$ reconstruction of a fractured femur

\section{Conclusions}

$>$ Mdk is involved in OVX-induced compromised fracture healing

$>$ Accelerated healing after Mdk-Ab treatment

$>$ Increased bone mass after Mdk-Ab treatment (callus and skeleton)
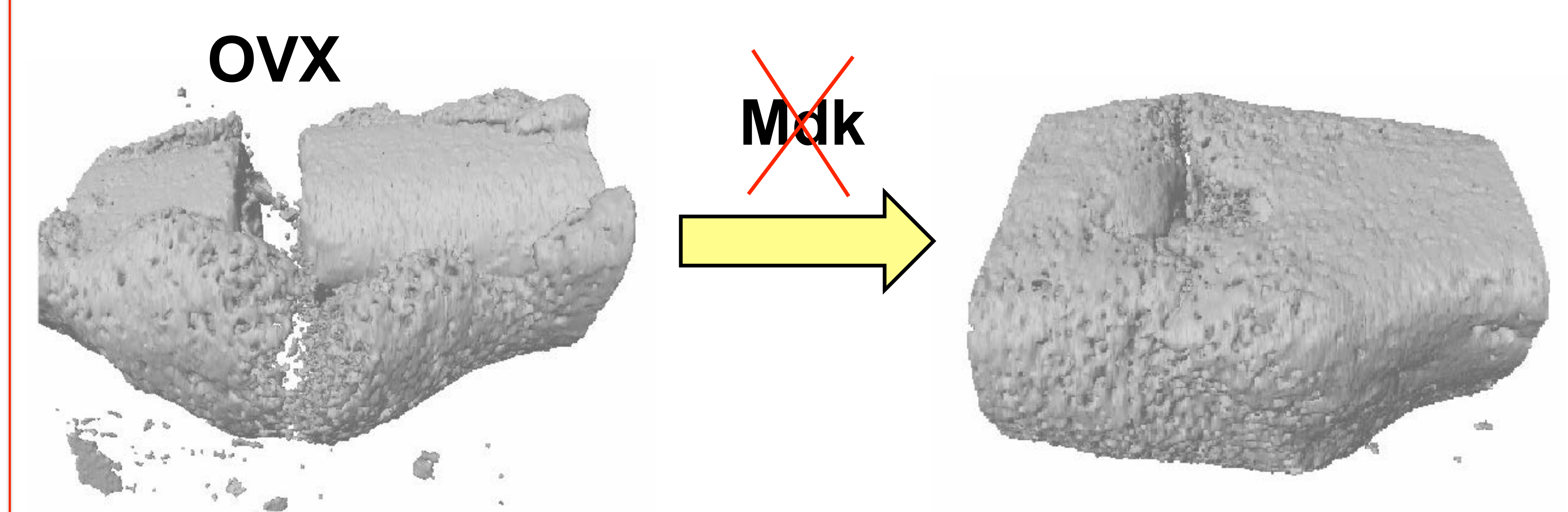

\section{Results}

Increased Mdk serum levels after fracture in OVX mice (Table 1):

$>$ fracture-induced increase of Mdk in the serum of sham-operated mice at day 3

$>$ significantly higher and prolonged expression of Mdk in the serum of OVX mice

$>$ significantly decreased Mdk serum levels after Mdk-Ab treatment

\begin{tabular}{|c|c|c|c|c|}
\hline & \multicolumn{4}{|c|}{ treatment } \\
\hline & \multicolumn{2}{|c|}{ sham } & \multicolumn{2}{|c|}{ OVX } \\
\hline $\begin{array}{l}\text { days after } \\
\text { operation }\end{array}$ & vehicle & Mdk-Ab & vehicle & Mdk-Ab \\
\hline $\mathrm{d} 0$ & \multicolumn{2}{|c|}{ n.d. } & \multicolumn{2}{|c|}{ n.d. } \\
\hline $\mathrm{d} 3$ & $38.6 \pm 44.6$ & $15.1 \pm 33.9$ & $67.9 \pm 45.7$ & $37.8 \pm 43.6$ \\
\hline$\overline{\mathrm{d} 10}$ & n.d. & n.d. & $61.7 \pm 43.6^{\mathrm{a}}$ & n.d. ${ }^{b}$ \\
\hline $\mathrm{d} 23$ & $\overline{\text { n.d. }}$ & n.d. & $31.0 \pm 28.2$ & n.d. \\
\hline
\end{tabular}

Table 1: Mdk serum levels in $\mathrm{pg} / \mathrm{ml}$ during bone healing.

Antagonizing Mdk abolished OVX-induced impaired healing:

$>$ OVX compromised fracture healing by decreased biomechanical competence and bone formation in the fracture callus

$>$ accelerated fracture healing after Mdk-Ab treatment in OVX mice

$>$ beta-catenin expression is regulated by $\operatorname{OVX}(\boldsymbol{\downarrow})$ and Mdk-Ab ( $\boldsymbol{\uparrow})$

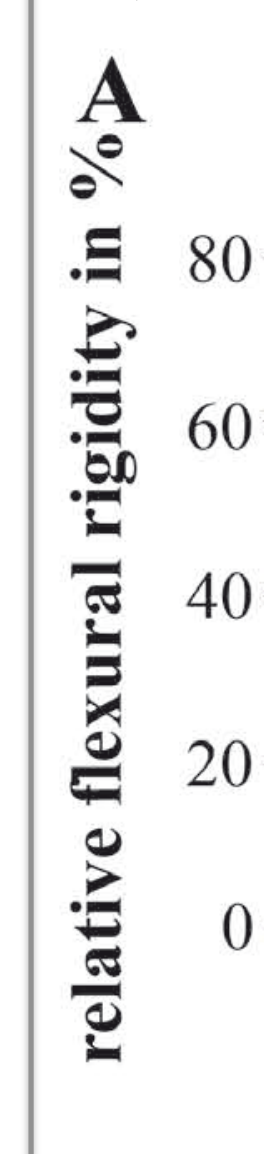

D
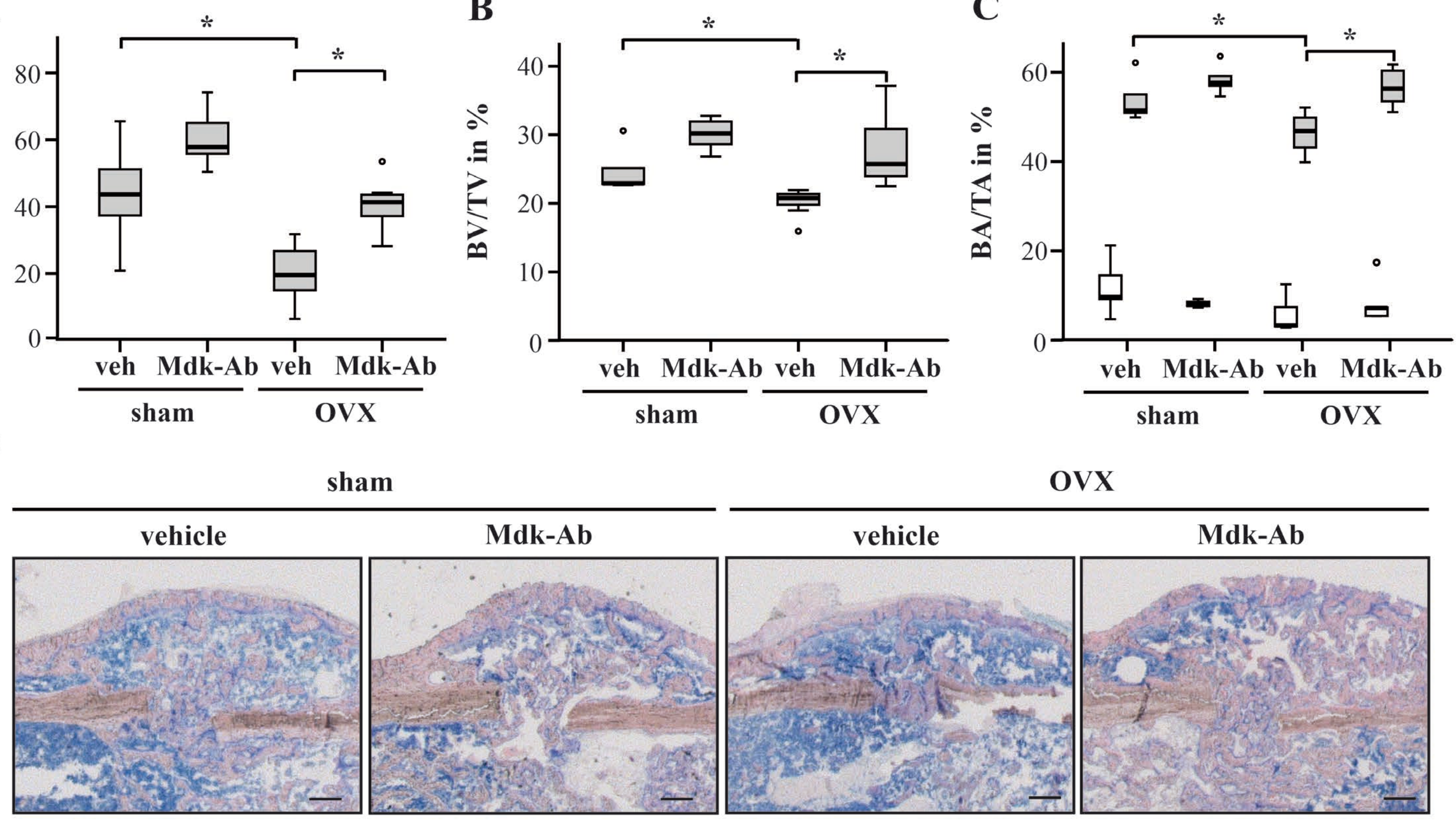

Fig. 2: A) Biomechanical competence of the fracture callus relative to the intact femur, day 23 B) $\mu C T$ analysis: bone volume to tissue volume ratio, day 23 C) Histology: Relative amount of bone in the fracture callus, white bars: day 10; grey bars: day 23. D) Callus sections from day 23, stained with Giemsa.

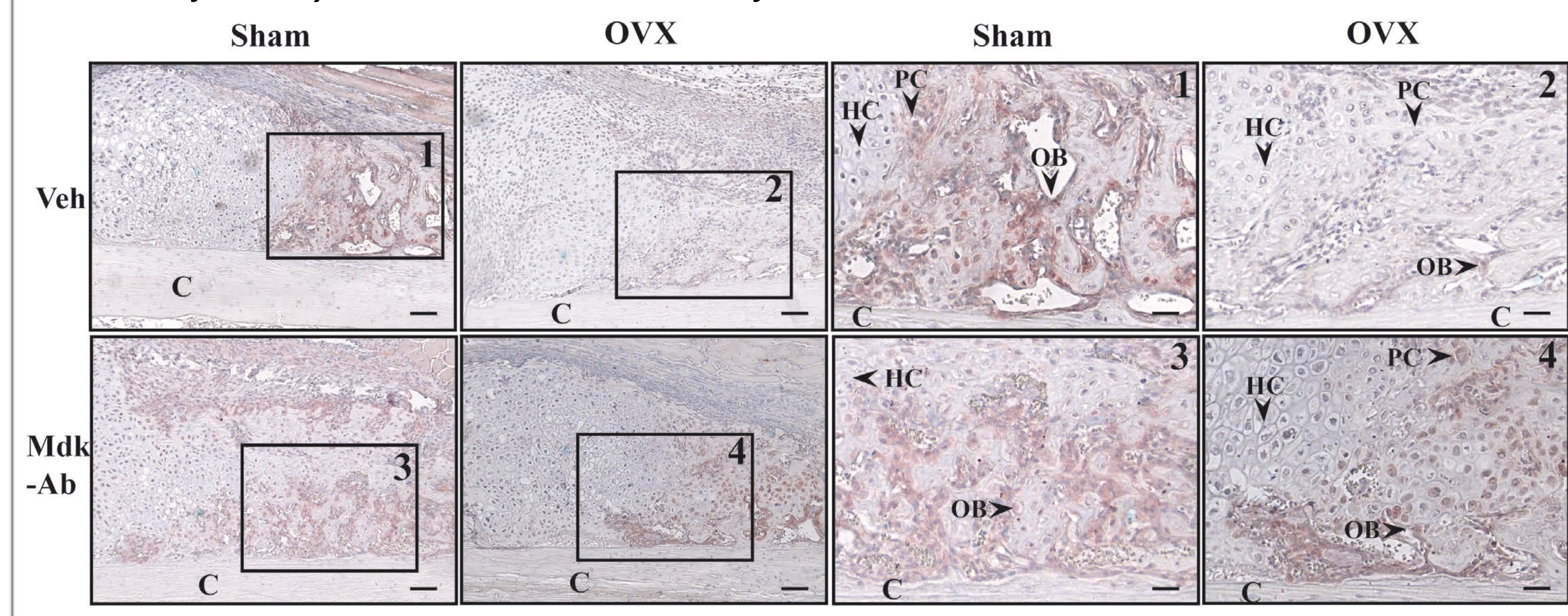

Fig. 3: Immunohistochemical staining for beta-catenin in the fracture callus at day 10. Increased bone mass in the intact skeleton after Mdk-Ab treatment:

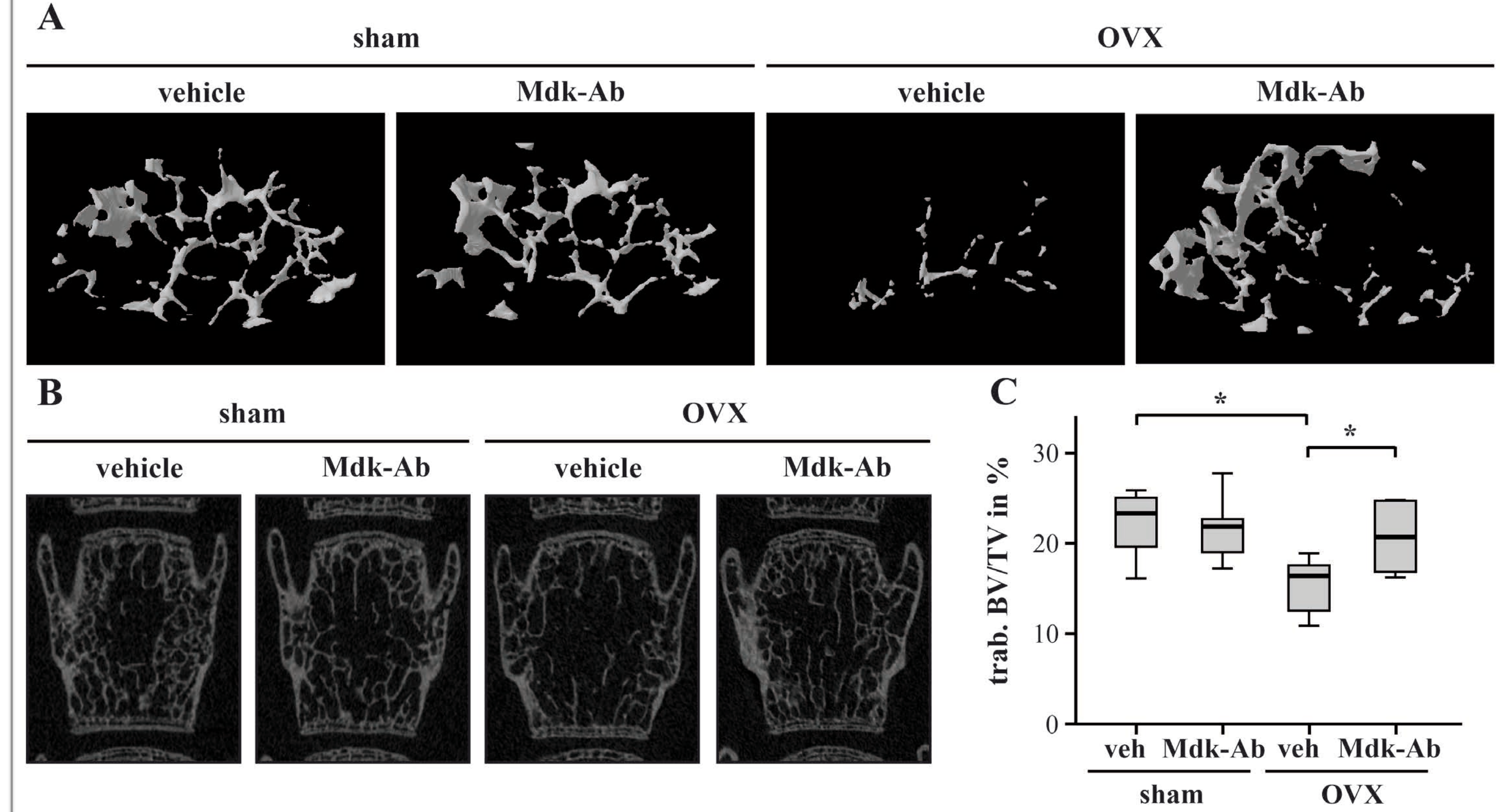

Fig. 4: A) $\mu C T 3 D$ recons from the intact femur metaphysis $B) \mu C T$ slices from the vertebral body $L 4$ C) Trabecular bone volume of $L 4$; all from day 23. 